\title{
IIIIII Hypoglycemia is More Common Among Type 2 Diabetes Patients with Limited Health Literacy: The Diabetes Study of Northern California (DISTANCE)
}

\author{
Urmimala Sarkar, MD, MPH ${ }^{1,2,6}$, Andrew J. Karter, $P h D^{3,4}$, Jennifer Y. Liu, MPH \\ Howard H. Moffet, MPH ${ }^{3}$, Nancy E. Adler, PhD $D^{5}$, and Dean Schillinger, MD ${ }^{1,2}$
}

'UCSF Center for Vulnerable Populations, San Francisco General Hospital, San Francisco, CA, USA; ${ }^{2}$ UCSF Division of General Internal Medicine, San Francisco General Hospital, San Francisco, CA, USA; ${ }^{3}$ Division of Research, Kaiser Permanente Northern California, Oakland, CA, USA; ${ }^{4}$ School of Public Health \& Community Health, University of Washington, Seattle, WA, USA; 5 UCSF Center for Health and Community, San Francisco, CA, USA; 'Department of Medicine, Division of General Internal Medicine, University of California, San Francisco, San Francisco, CA, USA.

BACKGROUND: Little is known about the frequency of significant hypoglycemic events in actual practice. Limited health literacy (HL) is common among patients with type 2 diabetes, may impede diabetes self-management, and thus HL could increase the risk of hypoglycemia.

OBJECTIVE: To determine the proportion of ambulatory, pharmacologically-treated patients with type 2 diabetes reporting $\geq 1$ significant hypoglycemic events in the prior 12 months, and evaluate whether HL is associated with hypoglycemia.

RESEARCH DESIGN: Cross-sectional analysis in an observational cohort, the Diabetes Study of Northern California (DISTANCE).

SUBJECTS: The subjects comprised 14,357 adults with pharmacologically-treated, type 2 diabetes who are seen at Kaiser Permanente Northern California (KPNC), a nonprofit, integrated health care delivery system.

MEASURES: Patient-reported frequency of significant hypoglycemia (losing consciousness or requiring outside assistance); patient-reported health literacy.

RESULTS: At least one significant hypoglycemic episode in the prior 12 months was reported by $11 \%$ of patients, with the highest risk for those on insulin (59\%). Patients commonly reported limited health literacy: 53\% reported problems learning about health, 40\% needed help reading health materials, and $32 \%$ were not confident filling out medical forms by themselves. After adjustment, problems learning (OR 1.4, CI 1.1-1.7), needing help reading (OR 1.3, CI 1.1-1.6), and lack of confidence with forms (OR 1.3, CI 1.1-1.6) were independently associated with significant hypoglycemia.

CONCLUSIONS: Significant hypoglycemia was a frequent complication in this cohort of type 2 diabetes

Funds were provided by National Institute of Diabetes, Digestive and Kidney Diseases RO1 DK65664 and National Institute of Child Health and Human Development RO1 HD046113. This work was also supported by the National Center for Research Resources. (KL2 RR024130).

Electronic supplementary material The online version of this article (doi:10.1007/s11606-010-1389-7) contains supplementary material, which is available to authorized users.

Received October 12, 2009

Revised March 13, 2010

Accepted April 20, 2010

Published online May 18, 2010 patients using anti-hyperglycemic therapies; those reporting limited HL were especially vulnerable. Efforts to reduce hypoglycemia and promote patient safety may require self-management support that is appropriate for those with limited HL, and consider more vigilant surveillance, conservative glycemic targets or avoidance of the most hypoglycemia-inducing medications.

KEY WORDS: diabetes; health literacy; hypoglycemia.

$\mathrm{J}$ Gen Intern Med 25(9):962-8

DOI: $10.1007 / \mathrm{s} 11606-010-1389-7$

(C) The Author(s) 2010. This article is published with open access at Springerlink.com

\section{INTRODUCTION}

Maintaining optimal glycemic control for type 2 diabetes has become a significant goal for health systems, providers, and patients. Landmark studies have shown that lowering blood glucose over the long term reduces microvascular complications, ${ }^{1,2}$. However, unexpected excess mortality recently reported in the ACCORD Trial, a randomized trial exploring the effects of lowering blood glucose to nearnormal levels, has renewed the controversy about glycemic targets $^{3-6}$ and raised important questions regarding treatment safety. One unintended consequence of optimizing glycemic control may be an increased risk for hypoglycemic events $^{3}$ (Fig. 1).

Hypoglycemia is one of the most common adverse drug events that lead to emergency room visits and hospitalization, ${ }^{7,8}$ and reduces quality of life $\mathrm{e}^{9,10}$. Although trials to assess the benefits of optimizing glycemic control among type 2 diabetes patients have documented a low incidence of hypoglycemia, ${ }^{11,12}$ hypoglycemia may be more common in practice $^{13}$, and little is known about the incidence of hypoglycemia occurrences that do not come to medical attention.

The relationship between limited health literacy and hypoglycemia is unclear. Limited health literacy (HL) has been found to be common among patients with type 2 diabetes ${ }^{14,15}$ and has been shown to be a barrier to adequate self-management of medication regimens, with less comprehension of medication instructions, dosing, timing, and 


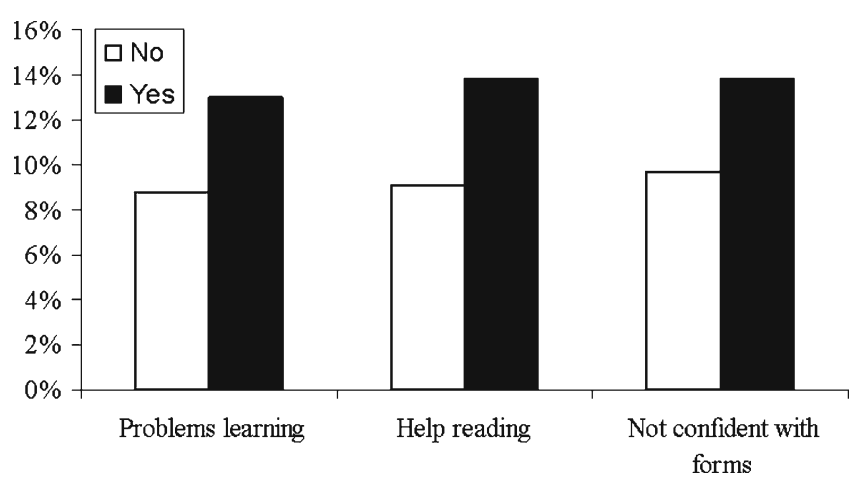

Figure 1. Proportion of patients reporting 1 or more significant hypoglycemic events in past 12 months.

warnings ${ }^{16-18}$, which could lead to increased risk for hypoglycemia.

We measured the occurrence of patient-reported significant hypoglycemia in a large, diverse population of medication-treated patients with type 2 diabetes with uniform access to care in an integrated managed care setting. We hypothesized that adults with diabetes would report significant hypoglycemia more often in this observational cohort than previously reported in clinical trials. We hypothesized that even after adjustment for a host of potential confounders, limited health literacy would be associated with hypoglycemia in an observational study of diabetes patients.

\section{METHODS}

\section{Study Population}

The Diabetes Study of Northern California (DISTANCE) enrolled an ethnically-stratified, random sample of patients from the Kaiser Permanente Northern California (KPNC) Diabetes Registry, a large, ethnically diverse and well-characterized population with diabetes. KPNC is a fully integrated health care delivery system that provides comprehensive medical care to over 3 million members $(30 \%$ of the entire Northern California population). Except for the extremes of income, the demographic characteristics of KPNC's patient population are similar to those of the overall population of Northern California ${ }^{19}$.

The overarching aim of the DISTANCE study (available at http://distancesurvey.org) was to investigate ethnic and educational disparities in diabetes-related behaviors, processes of care and health outcomes. The survey methods and cohort profile have been described previously ${ }^{20}$. The extensive survey was conducted among an ethnically-stratified, random sample of 40,735 diabetes patients, aged 30-75 at baseline, of known (Caucasian, African-American, Latino, Asian) and unknown ethnicity. Demographic, clinical, health care utilization and census data were obtained from electronic (non-survey) databases for the entire cohort. Briefly, three modes of the DISTANCE Survey were offered to all patients in the sample: self-administered written questionnaire, web-based survey, or computer-assisted telephone interview (CATI) with interviews conducted in English, Spanish, Cantonese, Mandarin or Tagalog.
Surveys were completed by 20,188 patients $(62 \%$ response rate, according to AAPOR response rate $\# 3$ definition $^{21}$ ). Respondents were primarily (78\%) of minority (non-White) race/ethnicity and varied widely in clinical and behavioral profiles, as well as education, income, wealth, occupation, nativity and neighborhood characteristics. For this analysis, we restricted our sample to respondents with type 2 diabetes (classified using a published algorithm ${ }^{22}$ ), since the incidence and mechanism of hypoglycemia may differ from type 1 diabetes ${ }^{23}$. We included only respondents taking any hyperglycemic medications, since strictly diet-controlled patients are generally not at risk for hypoglycemia ${ }^{24}$. We were able to obtain complete laboratory, prescription, emergency department and hospital utilization data from Kaiser's electronic records. This study was approved by the institutional review boards at Kaiser Foundation Research Institute.

\section{Measures.}

Outcome. We asked participants, "In the past year, how many times have you had a SEVERE low blood sugar reaction, such as passing out or needing help to treat the reaction?" This item was drawn from the widely-used, well-validated Diabetes Care Profile $^{25-27}$, and is similar to the hypoglycemia reporting items used in landmark diabetes trials and observational studies to identify treatment-related adverse events ${ }^{1,11-13,28}$. Responses were dichotomized into one or more episodes versus no episode. We considered "don't know" responses as missing.

Exposure. To obtain self-reported HL, we employed a slightly modified version of a 3 -item instrument that asks patients to report problems due to reading, understanding, and filling out forms, not due to poor vision: (a)"How often do you have problems learning about your medical condition because of difficulty understanding written information?"; (b) "How confident are you filling out medical forms by yourself?"; (c) "How often do you have someone like a family member, friend, hospital or clinic worker or caregiver, help you read Kaiser health plan materials (such as written information about your health or care you are offered)?" This instrument has been validated against direct $\mathrm{HL}$ measures ${ }^{29,30}$ in patients with diabetes and across a variety of research settings ${ }^{30-32}$. In keeping with prior studies, we dichotomized Likert responses into ever having difficulties versus never having difficulties ${ }^{30}$. We considered "don't know" responses as missing. Each question was analyzed separately, as the item authors recommend $^{29,30}$.

Analysis. First, we determined the frequency of reporting one or more episodes of significant hypoglycemia in the overall sample of pharmacologically treated patients with type 2 diabetes, then calculated its frequency across HL levels. Second, we performed an unadjusted logistic regression by regressing significant hypoglycemia on each HL question individually. Third, we performed three separate multivariate logistic regression analyses to assess the possible association of each HL item with hypoglycemia, adjusted for the demographic and clinical characteristics listed below and in 
Table 1. Because we used a stratified random sample (with over-sampling of racial/ ethnic minority patients), we needed to account for design effects by applying expansion weights (reciprocal of the non-proportional sampling fractions for each race) to multivariate models.

The multivariable analysis included age, sex, race/ethnicity, non-English language, and household income. Because hypoglycemia may be more common as renal function declines, we also adjusted for the glomerular filtration rate (GFR) using the MDRD equation ${ }^{33}$. We further adjusted for diabetes duration, hemoglobin Alc (HbAlc), and whether patients perform self-monitoring of blood glucose (SMBG), based on a validated measure constructed from prescription refill rates of glucose test strips ${ }^{22}$. We used diagnostic codes to adjust for cognitive impairment and dementia (ICD9 2900x, 29020, 2904x, 2941x, 2948x, 2949x, 3310x, 3311x, 33182x, 33183, 78093x), and past history of stroke (ICD9 431.x, 433.x, 434.x, 436.x). Finally, because some medication regimens are known to carry higher risk of hypoglycemia than others, we categorized participants' medication use, using pharmacy prescription data over the 12 months prior to the survey, as follows: (a) insulin alone or in combination with oral agents; (b) oral secretagogues (such as sulfonylureas) alone; (c) metformin alone; (d) mixed oral agents, and adjusted for type of regimen in all analyses. We also explored the possible contribution of body-mass index, problematic alcohol use, medication adherence (continuous medication gap ${ }^{34}$ ), and neuropathy (ICD 9 250.6x) in additional multivariable models.

Table 1. Subject Characteristics $(N=14,357)$

\begin{tabular}{|c|c|}
\hline Characteristics & $\mathrm{N}$ or mean $(\%$ or $\mathrm{SD})$ \\
\hline Age (y) & $58(10)$ \\
\hline Female sex & $7,068(49)$ \\
\hline \multicolumn{2}{|l|}{ Race/ Ethnicity } \\
\hline African-American & $2,417(17)$ \\
\hline Non-Hispanic White & $3,202(22)$ \\
\hline Latino/a & $2,632(18)$ \\
\hline Asian & $3,265(23)$ \\
\hline Other/Mixed & $2,841(20)$ \\
\hline Limited English Proficiency & $1,386(10)$ \\
\hline $\mathrm{HbAlc} \%$ & $7.6(1.6)$ \\
\hline \multicolumn{2}{|l|}{ Medication Type } \\
\hline Insulin & $3,142(22)$ \\
\hline Secretagogues only & $2,284(16)$ \\
\hline Metformin only & $2,727(19)$ \\
\hline Mixed Oral Meds & $6,205(43)$ \\
\hline Diabetes duration, yrs & $10(8)$ \\
\hline Perform self-monitoring of blood glucose & $6,934(48)$ \\
\hline Problems learning & $7,617(53)$ \\
\hline Help reading & $5,726(40)$ \\
\hline Not confident with forms & $4,539(32)$ \\
\hline Dementia & $159(1)$ \\
\hline Cerebrovascular disease/ stroke & $382(3)$ \\
\hline \multicolumn{2}{|l|}{ Renal function } \\
\hline GFR $>=90$ & $2,087(15)$ \\
\hline GFR 60-89 & 7,069 (49) \\
\hline GFR 30-59 & $3,037(21)$ \\
\hline GFR 15-29 & $219(2)$ \\
\hline GFR $<15$ & $135(1)$ \\
\hline \multicolumn{2}{|l|}{ Income } \\
\hline$>\$ 65,000$ & $4,673(33)$ \\
\hline$\$ 35,000-\$ 65,000$ & $3,728(26)$ \\
\hline$\$ 25,000-\$ 34,999$ & $1,472(10)$ \\
\hline$\$ 15,000-\$ 24,999$ & $1,080(8)$ \\
\hline$<\$ 15,000$ & $1,305(9)$ \\
\hline
\end{tabular}

We wished to address 2 types of response bias: survey nonresponse and item non-response. For survey non-response, we used the standard Horvitz-Thompson ${ }^{35}$ approach. This multipart model, first uses available data to calculate the probability of survey response. The inverse of that fitted non-response probability is then used to weight the complete observations. Even among survey respondents, not all answered all questions in the 52-page survey, leading to some missing values for outcome, exposure (HL), and co-variates. Item nonresponse is a common problem in lengthy surveys. Analysis restricted to complete cases can introduce bias if the data are not missing completely at random, and also reduce precision $^{36}$. Therefore, we used multiple imputation, as implemented in SAS Proc $\mathrm{MI}^{37}$. Missing items were imputed ten times under the assumption that they were missing, not completely at random, but at random, given the covariates in the imputation models; then the ten completed datasets were each analyzed using complete data methods, and the results combined using established methods that provide standard errors, confidence intervals and $\mathrm{p}$-values that properly reflect the imputation of the missing items. Imputed values for binary and categorical variables were rounded and truncated to the nearest category ${ }^{38}$. Final parameter estimates, confidence intervals, and significance tests were calculated using standard methods for combining results across the ten imputed data sets ${ }^{38,39}$, as implemented in Proc MIANALYZE. In addition, we performed a sensitivity analyses using only complete cases, those for whom no data were missing. Finally, we examined whether the sample included in the current analysis (those with pharmacologically-treated type 2 diabetes), exhibited demographic or clinical differences compared to the survey respondents overall.

Because there may be underreporting of limited HL due to shame ${ }^{16}$, we performed a sensitivity analysis in which we assumed that those with "don't know" responses to the HL items had limited HL. We also explored hypothesized interactions between HL and potential effect modifiers including age, diabetes medication treatment, and survey mode by adding cross-product terms to the model specified above. To further evaluate the possibility that inaccurate reporting of hypoglycemia contributed to our findings, we performed a sensitivity analysis involving only the subgroup (94\%) that provided a correct answer to a hypoglycemia knowledge item from the DISTANCE survey that asked "If a person with diabetes suddenly gets sweaty, nervous, and shaky, what should he or she do?" Finally, as an exploratory analysis to further validate the outcome assessment, we compared the odds of participants' making an emergency department visit or requiring a hospitalization for hypoglycemia (International Classification of Disease Codes $250.8,251.0,251.1,251.2$ ) over the 3 -year period prior to survey completion between those who did versus did not report significant hypoglycemia in the survey.

\section{RESULTS}

Of the 20,188 surveyed, 2,393 were excluded because they completed a short-form of the survey which lacked the HL items. We further excluded 826 because they did not have type 2 diabetes, and 2612 who did not take medication for type 2 
diabetes. Thus, our overall sample was the 14,357 responders with pharmacologically treated type 2 diabetes whom we asked about health literacy. Subjects were ethnically-diverse, had long-standing diabetes (average time since diagnosis approximately 10 years) and relatively well-controlled glycemia (average HbAlc of 7.6) (Table 1). The most common medication regimen was mixed oral agents (43\%), followed by insulin alone or in combination with oral agents $(22 \%)$, metformin alone (19\%), and secretagogues alone (16\%). According to refill rate of glucose test strips, $48 \%$ regularly self-monitor their blood glucose (SMBG). Among all respondents, 53\% reported problems learning about health, $40 \%$ needed help reading healthcare materials, and $32 \%$ were not confident with medical forms, due to reading difficulties. We did not observe clinically meaningful differences between the 14,357 we analyzed and the 20,188 survey respondents (see online appendix Table 1).

Overall, 1,579 (11\%) of participants reported experiencing at least one significant hypoglycemic episode in the past year. Hypoglycemia occurrence varied by medication regimen, with the highest risk for those on insulin (59\%) followed by, mixed oral medication types (23\%), secretagogues alone (13\%) and metformin alone (5\%). Of these participants who reported at least 1 significant hypoglycemic episode, 8\% (129/1579) had evidence of a documented emergency department visit or hospitalization for hypoglycemia in the prior year, compared to $1.6 \%(202 / 12,777)$ of the participants who reported not having significant hypoglycemia, yielding an odds ratio of 19.0 (95\% CI 13.0-26.0).

A significantly higher proportion of patients reporting limited HL experienced significant hypoglycemia ( $\mathrm{p}$ for all $<0.0001$; Figure). This pattern was consistent across all three HL questions, with each accompanying analysis yielding very similar effect sizes (Table 2). These relationships did not change substantively with adjustment for patient factors (age, sex, race/ethnicity, language, income, social support, diabetes factors (glycemic control, diabetes duration, treatment regimen, SMBG practice), and renal function. In additional models further adjusted for body-mass index, alcohol use, medication adherence, and neuropathy, the relationship between HL and hypoglycemia remained unchanged (see online appendix Table 2.) While we found that those with limited HL were more likely than those with adequate HL to respond by CATI than either web or mailed written surveys ( $p$ for all $<0.001$ ), the relationship between HL and hypoglycemia did not differ significantly across survey modes (p-value for all HL-survey $\backslash$ mode cross-product terms >0.2). In stratified analyses,

Table 2. Unadjusted and Adjusted Odds Ratios of Experiencing Significant Hypoglycemia (Versus Not) by Self-reported HL

\begin{tabular}{llll}
\hline \hline & \multicolumn{1}{l}{ Unadjusted } & & Adjusted $^{\mathrm{a}}$ \\
\cline { 2 - 2 } & Odds Ratio $(95 \% \mathrm{Cl})$ & & Odds Ratio (95\% Cl) \\
\hline $\begin{array}{lll}\text { Problems learning } \\
\text { Need help reading }\end{array}$ & $1.5(1.3-1.8)$ & & $1.4(1.1-1.7)$ \\
$\begin{array}{l}\text { Not confident with } \\
\text { forms }\end{array}$ & $1.5(1.3-1.8)$ & & $1.3(1.1-1.6)$ \\
& & & $1.3(1.1-1.6)$ \\
\hline
\end{tabular}

aAdjusted for age, gender, race/ethnicity, English proficiency, medication type, diabetes duration, HbAlc, glomerular filtration rate, income, dementia and history of stroke. neither age nor medication type substantively or significantly modified the HL-hypoglycemia relationships. Results did not differ substantively in the complete-case only analysis. In a sensitivity analysis that excluded all participants who did not demonstrate knowledge of hypoglycemia symptoms and treatment, results remained substantively unchanged. When we classified participants who responded "don't know" to the health literacy questions as limited health literacy, the results did not change. Finally, our results were substantively unchanged in an analysis re-weighted for the inverse probability of survey response (data not shown).

\section{CONCLUSIONS}

Among ambulatory type 2 diabetes patients receiving care in a well-integrated, managed-care system, we found that hypoglycemia is a significant problem. To our knowledge, this is the largest observational study in the U.S. to assess significant hypoglycemia. As prior studies would suggest ${ }^{40}$, the frequency of significant hypoglycemia reported in this population was significantly higher than reported in landmark trials. Annual assistance-requiring hypoglycemia rates range from 0.7 per 100 overall for $\mathrm{ADVANCE}^{1}$ and $5.1 \%$ in the standard arm for $\mathrm{ACCORD}^{41}$. In UKPDS, the highest rate of assistance-requiring hypoglycemia was among insulin users in the intensive arm, and was only $1.8 \% /$ year $^{1}$. The prevalence of self-reported hypoglycemia relative to emergency department visits or hospitalizations coded for hypoglycemia suggests that (a) the vast majority of events are cared for outside of the medical setting, (b) clinicians and health systems may not fully appreciate the scope of this problem and that (c) most patients are choosing to self-manage any immediate consequences of hypoglycemia without medical assistance. Whether patients are developing and implementing strategies to avoid future hypoglycemia events on their own, or in consultation with their treating clinicians, is not known.

Hypoglycemia, while an important adverse event in and of itself, contributes to poorer diabetes outcomes in other domains. Recent work has shown that acute care visits for hypoglycemia predict future risk for dementia among type 2 diabetes patients ${ }^{42}$. Many have suggested that those who experience hypoglycemia may have lower rates of hypoglycemic medication adherence for fear of recurrence ${ }^{43,44}$.

Significant hypoglycemia was especially common among patients reporting limited HL, and this association persisted even after adjusting for known and suspected correlates of HL and hypoglycemia. There are several possible mechanisms by which limited HL could increase hypoglycemia risk. First, limited HL could lead to patient errors in timing or dosage of diabetes medications ${ }^{16,45}$. Second, limited HL could interfere with proper adjustment of diabetes medication in response to changing caloric intake or energy expenditure ${ }^{46}$. Third, those with limited HL may not correctly interpret and respond to their blood glucose values and trends obtained through self-monitoring, potentially missing opportunities to adapt self-management behaviors so as to prevent significant hypoglycemia ${ }^{47}$. Importantly, mastery of such self-management skills depends, in part, on adequate patient-clinician communication. Limited HL has been shown to impede both 
patients' understanding of clinician information and instructions, and patients' reporting of symptoms and disease state to clinicians ${ }^{18}$.

Study strengths include a large, diverse population with uniform access to care, and detailed assessments of HL and hypoglycemic events within a real world setting. Nevertheless, this study has several limitations. First, the KPNC system provides coordinated and uniform access to care, including diabetes education and comprehensive care management. As such, our results may under-estimate hypoglycemic event rates in the under- and uninsured, ${ }^{48}$ or among populations cared for in non-integrated health systems. Second, because this is a cross-sectional analysis, we cannot infer that limited HL was the cause of increased hypoglycemia risk; although reverse causation is improbable, there may be underlying factors affecting both. Third, we did not directly measure HL, although the self-report HL items we used have been validated against direct measurement instruments ${ }^{29,30}$. Moreover, the degree to which these items exactly correlate with HL as assessed by standard, 'direct' measurement may be less relevant in practice, insofar as these questions have predictive validity as they appear to identify subgroups at risk for a clinically important adverse event. Fourth, we did not measure numeracy in this study, so we could not address the relative contribution of numeracy versus literacy. Numeracy and literacy have been shown to be highly correlated. Fifth, we could not capture attendance at diabetes education. Sixth, not all participants answered every question in this lengthy survey (184 questions, 52 pages). Nevertheless, this remains the largest observational study of hypoglycemia, and the subjects included in the analysis did not differ from the overall sample in terms of age, race/ ethnicity, educational attainment, which are the best predictors of health literacy, or HbAlc, renal function, or medication type, the clearest correlates of hypoglycemia. In addition, we used multiple imputation to minimize the bias associated with missing items $^{36,38,39,49}$. Moreover, results using multiply imputed data did not differ from the complete data-only approach. Seventh, we could not explore individual medications within each class. Finally, consistent with prior studies, ${ }^{1,10-13,50}$ we obtained measures of hypoglycemia via patient self-report, albeit an imperfect measure. Since self-report of mild hypoglycemia may be less accurate, we queried only clinically significant episodes of losing consciousness or requiring treatment assistance. The fact that we observed a stepwise increase in hypoglycemia consistent with the established pharmacologic risks of hypoglycemia from different medication regimens (i.e., highest in insulin-treated patients) suggests the validity of patients' reports. We also found that self-report of hypoglycemia was strongly associated with likelihood of making an emergency room visit or requiring hospitalization for hypoglycemia during the year prior to the survey, providing additional validation of this self-reported measure. Furthermore, while it is possible that those with limited HL were more likely to misattribute non-hypoglycemic events to hypoglycemia, the results of our sensitivity analysis that involved only those participants who demonstrated accurate knowledge of hypoglycemia symptoms and treatment suggest that outcome misclassification does not explain our findings. It is theoretically possible that those with limited HL reported more significant hypoglycemia because they may require help for hypoglycemic episodes while those with adequate HL can manage themselves. Such a heightened need for assistance would, in and of itself, place them at greater risk.

Our findings have several clinical and health policy implications. First, while it is unclear whether treating clinicians' actions (such as intensifying medications or inadequate communication of safety precautions) contributed to these events or whether treating clinicians were aware of patients' hypoglycemic episodes, our results suggest that hypoglycemic symptoms should be routinely assessed in patients with type 2 diabetes receiving hyperglycemia-lowering agents. Second, because insulin use confers a particularly high risk for hypoglycemia, we recommend formal hypoglycemia prevention counseling that is appropriate for those with limited health literacy, as well as more vigilant self-management support that includes training family members and caregivers, at the time of insulin initiation. More research is needed to (a) identify whether errors in patient self-management are contributing to hypoglycemia, and (b) determine what actions by patients, families, caregivers and clinicians can reduce hypoglycemia. Third, hypoglycemia risk profile should be an important criteria when choosing medication regimen, favoring medications which are least likely to cause hypoglycemia.

Hypoglycemia has been described as a sentinel adverse event ${ }^{7}$ in type 2 diabetes that should trigger systems-based approach to reduce recurrences. System-level surveillance for hypoglycemia, perhaps through use of patient-directed health information technology, may promote patient safety. Between-visit surveillance mechanisms, utilizing technologies such as interactive voice response technology, ${ }^{30,5,52}$ personal health internet portals, ${ }^{53-55}$ or continuous blood glucose monitors ${ }^{56,57}$ may be more sensitive in capturing hypoglycemia than are current practices in diabetes management. For example, patients receiving periodic automated telephone self-management support for diabetes could input a low blood glucose value into their telephone touchpad, which would lead to a live telephone call with a care manager as well as notification to the primary care provider. Such technologies have the potential to make use of visual and voice modalities so as to be appropriate for those with limited HL, but this requires further investigation. Finally, from a health policy perspective, our results suggest higher rates of hypoglycemia than previously documented, raising the question of whether widely-recommended clinical targets and performance measures derived from efficacy studies, when applied to real world contexts, may jeopardize the safety of many patients with type 2 diabetes $^{3,4}$.

Acknowledgements: Authors (AJK, HHM, JYL) had full access to all of the data in the study and take responsibility for the integrity of the data and the accuracy of the data analysis. Funds were provided by National Institute of Diabetes, Digestive and Kidney Diseases RO1 DK65664 and National Institute of Child Health and Human Development RO1 HD046113. Dr. Sarkar is supported by Agency for Healthcare Research and Quality K08 HSO17594. Dr. Schillinger is supported by a grant from Agency for Healthcare Research and Quality R18 HSO1726101 a NIH Clinical and Translational Science Award ULRR024131. None of the funders had any role in design and 
conduct of the study; collection, management, analysis, or interpretation of the data; or preparation, review, or approval of the manuscript. We gratefully acknowledge Andrea Lopez's assistance in preparing the revised manuscript.

Conflicts of Interest: Dr. Sarkar is supported by Agency for Healthcare Research and Quality K08 HSO17594. Dr. Schillinger is supported by a grant from Agency for Healthcare Research and Guality R18 HSO1726101 a NIH Clinical and Translational Science Award ULRR024131. None of the funders had any role in design and conduct of the study; collection, management, analysis, or interpretation of the data; or preparation, review, or approval of the manuscript.

Open Access: This article is distributed under the terms of the Creative Commons Attribution Noncommercial License which permits any noncommercial use, distribution, and reproduction in any medium, provided the original author(s) and source are credited.

Corresponding Author: Urmimala Sarkar, MD, MPH; Department of Medicine, Division of General Internal Medicine, University of California, San Francisco, Box 1364, 1001 Potrero, Bldg 10, 3rd floor, San Francisco, CA 94143-1364, USA (e-mail: usarkar@medsfgh.ucsf. edu).

\section{REFERENCES}

1. UK Prospective Diabetes Study (UKPDS) Group. Intensive blood-glucose control with sulphonylureas or insulin compared with conventional treatment and risk of complications in patients with type 2 diabetes (UKPDS 33). Lancet. 1998;352(9131):837-53.

2. UK Prospective Diabetes Study (UKPDS) Group. Effect of intensive bloodglucose control with metformin on complications in overweight patients with type 2 diabetes (UKPDS 34). Lancet. 1998;352(9131):854-65.

3. Gerstein HC, Miller ME, Byington RP, et al. Effects of intensive glucose lowering in type 2 diabetes. N Engl J Med. 2008;358(24):2545-59.

4. Patel A, MacMahon S, Chalmers $\mathbf{J}$, et al. Intensive blood glucose control and vascular outcomes in patients with type 2 diabetes. $\mathrm{N}$ Engl $\mathrm{J}$ Med. 2008;358(24):2560-72.

5. Cefalu WT, Watson $\mathbf{K}$. Intensive glycemic control and cardiovascular disease observations from the ACCORD study: now what can a clinician possibly think? Diabetes. 2008;57(5):1163-5.

6. Ruo B, Rumsfeld JS, Hlatky MA, Liu H, Browner WS, Whooley MA. Depressive symptoms and health-related quality of life: the Heart and Soul Study. JAMA. 2003;290(2):215-21.

7. Budnitz DS, Pollock DA, Weidenbach KN, Mendelsohn AB, Schroeder TJ, Annest JL. National surveillance of emergency department visits for outpatient adverse drug events. JAMA. 2006;296(15):1858-66.

8. Budnitz DS, Shehab N, Kegler SR, Richards CL. Medication use leading to emergency department visits for adverse drug events in older adults. Ann Intern Med. 2007;147(11):755-65.

9. Lundkvist J, Berne C, Bolinder B, Jonsson L. The economic and quality of life impact of hypoglycemia. Eur J Health Econ. 2005;6(3): 197 202.

10. Spitzer RL, Kroenke K, Williams JB. Validation and utility of a selfreport version of PRIME-MD: the PHQ primary care study. Primary care evaluation of mental disorders. Patient health questionnaire. JAMA. 1999;282(18):1737-44.

11. Abraira C, Colwell JA, Nuttall Fg, et al. Veterans Affairs Cooperative Study on glycemic control and complications in type II diabetes (VA CSDM). Results of the feasibility trial. Veterans Affairs Cooperative Study in Type II Diabetes. Diabetes Care. 1995;18(8):1113-23.

12. Nathan DM, Roussell A, Godine JE. Glyburide or insulin for metabolic control in non-insulin-dependent diabetes mellitus. A randomized, double-blind study. Ann Intern Med. 1988;108(3):334-40.

13. Alvarez Guisasola F, Tofe Povedano S, Krishnarajah G, Lyu R, Mavros P, Yin D. Hypoglycaemic symptoms, treatment satisfaction, adherence and their associations with glycaemic goal in patients with type 2 diabetes mellitus: findings from the Real-Life Effectiveness and Care Patterns of Diabetes Management (RECAP-DM) Study. Diabetes Obes Metab. 2008;10(Suppl 1):25-32.
14. Morris NS, MacLean CD, Littenberg B. Literacy and health outcomes: a cross-sectional study in 1002 adults with diabetes. BMC Fam Pract. 2006;7:49.

15. Schillinger D, Piette J, Grumbach K, et al. Closing the loop: physician communication with diabetic patients who have low health literacy. Arch Intern Med. 2003;163(1):83-90.

16. Wolf MS, Williams MV, Parker RM, Parikh NS, Nowlan AW, Baker DW. Patients' shame and attitudes toward discussing the results of literacy screening. J Health Commun. 2007;12(8):721-32.

17. Weingart SN, Simchowitz B, Padolsky H, et al. An empirical model to estimate the potential impact of medication safety alerts on patient safety, health care utilization, and cost in ambulatory care. Arch Intern Med. 2009;169(16): 1465-73.

18. Sudore RL, Yaffe K, Satterfield S, et al. Limited literacy and mortality in the elderly: the health, aging, and body composition study. J Gen Intern Med. 2006;21(8):806-12.

19. Krieger N. Overcoming the absence of socioeconomic data in medical records: validation and application of a census-based methodology. Am J Public Health. 1992;82(5):703-10.

20. Sarkar U, Karter A, Adler N, Liu J, Moffet H, Schillinger D. Limited health literacy is associated with increased risk of hypoglycemia among insured, type 2 diabetes patients: the Diabetes Study of Northern California (DISTANCE) Society for General Internal Medicine. Pittsburgh, PA. J Gen Intern Med. 2008;23(s2):338.

21. AAPOR. Standard Definitions. Vol. February 12; 2009.

22. Selby JV, Uratsu CS, Fireman B, et al. Treatment intensification and risk factor control: toward more clinically relevant quality measures. Med Care. 2009;47(4):395-402.

23. Amiel S, Dixon T, Mann R, Jameson K. Hypoglycemia in Type 2 diabetes. Diabet Med. 2008;25(3):245-54.

24. Gardner D, Shobeck D. Greenspan's Basic and Clinical Endocrinology. 8th ed. New York: McGraw-Hill; 2007.

25. Fitzgerald ST, Zlotnick C, Kolodner KB. Factors related to functional status after percutaneous transluminal coronary angioplasty. Heart Lung. 1996;25(1):24-30.

26. Fitzgerald JT, Anderson RM, Gruppen LD, et al. The reliability of the Diabetes Care Profile for African Americans. Eval Health Prof. 1998;21(1):52-65.

27. Cunningham V, Mohler MJ, Wendel CS, et al. Reliability and validity of the DCP among hispanic veterans. Eval Health Prof. 2005;28(4):447-63.

28. Miller CD, Phillips LS, Ziemer DC, Gallina DL, Cook CB, El-Kebbi IM. Hypoglycemia in patients with type 2 diabetes mellitus. Arch Intern Med. 2001;161(13):1653-9.

29. Morris NS, MacLean CD, Chew LD, Littenberg B. The Single Item Literacy Screener: evaluation of a brief instrument to identify limited reading ability. BMC Fam Pract. 2006;7:21.

30. Sarkar U, Piette JD, Gonzales R, et al. Preferences for self-management support: findings from a survey of diabetes patients in safety-net health systems. Patient Educ Couns. 2008;70(1):102-10.

31. Wallace LS, Cassada DC, Rogers ES, et al. Can screening items identify surgery patients at risk of limited health literacy? J Surg Res. 2007;140 (2):208-13.

32. Wallace LS, Rogers ES, Roskos SE, Holiday DB, Weiss BD. Brief report: screening items to identify patients with limited health literacy skills. J Gen Intern Med. 2006;21(8):874-7.

33. Levey AS, Bosch JP, Lewis JB, Greene T, Rogers N, Roth D. A more accurate method to estimate glomerular filtration rate from serum creatinine: a new prediction equation. Modification of Diet in Renal Disease Study Group. Ann Intern Med. 1999;130(6):461-70.

34. Steiner JF, Koepsell TD, Fihn SD, Inui TS. A general method of compliance assessment using centralized pharmacy records. Description and validation. Med Care. 1988;26(8):814-23.

35. Horvitz DG, Source DJT. A Generalization of Sampling Without Replacement From a Finite Universe. J Am Stat Assoc. 1952;47:66385. American Statistical Association Stable Dec.1952.

36. Schafer J, Graham J. Missing data: our view of the state of the art Psychol Meth. 2002;7(2):147-77.

37. Schafer JL. Multiple imputation: a primer. Stat Methods Med Res. 1999;8(1):3-15.

38. Allison P. Missing Data. Thousand Oaks: Sage Publications; 2002.

39. Rubin D. Multiple imputation for nonresponse in surveys. New York: Wiley; 1987.

40. Cryer PE, Davis SN, Shamoon H. Hypoglycemia in diabetes. Diab Care. 2003;26(6): 1902-12.

41. Action to Control Cardiovascular Risk in Diabetes Study G, Gerstein HC, Miller ME, et al. Effects of intensive glucose lowering in type 2 diabetes. N Engl J Med. 2008;358(24):2545-59. 
42. Whitmer R, Karter A, Yaffe K, Quesenberry CJ, Selby J. Hypoglycemia episodes and risk of dementia in older patients with type 2 diabetes: findings from the diabetes and aging study. J Am Med Assoc. 2009;in press.

43. Davis S, Alonso MD. Hypoglycemia as a barrier to glycemic control. J Diabetes Its Complicat. 2004;18(1):60-8.

44. Cryer PE. Hypoglycaemia: the limiting factor in the glycaemic management of Type I and Type II diabetes. Diabetologia. 2002;45(7):937-48.

45. Persell SD, Osborn CY, Richard R, Skripkauskas S, Wolf MS. Limited health literacy is a barrier to medication reconciliation in ambulatory care. J Gen Intern Med. 2007;22(11):1523-6.

46. Paasche-Orlow MK, Wolf MS. The causal pathways linking health literacy to health outcomes. Am J Health Behav. 2007;31(Suppl 1):S19-26.

47. Cavanaugh $\mathbf{K}$, Huizinga MM, Wallston KA, et al. Association of numeracy and diabetes control. Ann Intern Med. 2008;148(10):737-46.

48. Nelson K, Brown ME, Lurie N. Hunger in an adult patient population. JAMA. 1998;279(15):1211-4.

49. Slymen DJ, Drew JA, Wright BL, Elder JP, Williams SJ. Item nonresponse to lifestyle assessment in an elderly cohort. Int $\mathrm{J}$ Epidemiol. 1994;23(3):583-91.

50. Ohkubo Y, Kishikawa H, Araki E, et al. Intensive insulin therapy prevents the progression of diabetic microvascular complications in Japanese patients with non-insulin-dependent diabetes mellitus: a randomized prospective 6-year study. Diabetes Res Clin Pract. 1995;28 (2): 103-17.
51. Alkema GE, Wilber KH, Shannon GR, Allen D. Reduced mortality: the unexpected impact of a telephone-based care management intervention for older adults in managed care. Health Serv Res. 2007;42 (4): 1632-50.

52. Classen DC, Kanhouwa M, Will D, Casper J, Lewin J, Walker J. The patient safety institute demonstration project: a model for implementing a local health information infrastructure. J Healthc Inf Manag. 2005; 19 (4):75-86.

53. Schoenthaler A, Chaplin WF, Allegrante JP, et al. Provider communication effects medication adherence in hypertensive African Americans. Patient Educ Couns. 2009;75(2):185-91.

54. Grant RW, Wald JS, Poon EG, et al. Design and implementation of a web-based patient portal linked to an ambulatory care electronic health record: patient gateway for diabetes collaborative care. Diabetes Technol Ther. 2006;8(5):576-86.

55. Hess R, Bryce CL, Paone S, et al. Exploring challenges and potentials of personal health records in diabetes self-management: implementation and initial assessment. Telemed J E Health. 2007;13(5):509-17.

56. Smits M, Groenewegen PP, Timmermans DR, van der Wal G, Wagner C. The nature and causes of unintended events reported at ten emergency departments. BMC Emerg Med. 2009;9:16.

57. McGarraugh G, Bergenstal R. Detection of hypoglycemia with continuous interstitial and traditional blood glucose monitoring using the freestyle navigator continuous glucose monitoring system. Diabetes Technol Ther. 2009 . 